\title{
GENERAL WAVE EQUATION IN THE ELECTROSTATIC APPROXIMATION
}

\author{
A. V. Agafonov \\ P. N. Lebedev Physical Institute, Leninsky Prosp. 53, Moscow 117924, Russia
}

\begin{abstract}
Equilibria of high-current electron beams in an axial magnetic field are considered at the base of the approach [1] for the two cases of shielded and immersed cathodes. A general wave equation in the electrostatic approximation, which is valid in any selfconsistent equilibrium, was derived under the only assumption of constancy of full particle energy. This equation supplements general steady state equation [1] and allows to consider different equilibria and their wave properties in closed manner.
\end{abstract}

\section{INTRODUCTION}

For proper investigation of wave processes in REB it is necessary to use models of beam equilibria that could be solved analytically and would be consistent with an experimental condition. Often, the analytical solvability of a model is beyond its conformity to the real conditions. A general wave equation for selfconsistent equilibria is also important as it permits to compare the influence of different cross terms that can be omitted because of approximate solution of the steady state problem. In view of space limitations only these two problems are discussed.

\section{LAMINAR FLOWS EQUILIBRIA}

In cylindrical coordinates $\mathrm{r}, \theta, \mathrm{z}$, under the conditions $\partial / \partial t=\partial / \partial z=\partial / \partial \theta=0$, steady state equilibria of a beam in the longitudinal magnetic field $B_{z 0}$ are described by the following equations

$$
\begin{array}{r}
\frac{\gamma v_{\theta}^{2}}{r}+E_{r}+v_{\theta} B_{z}-v_{z} B_{\theta}=0 ; \\
\frac{d B_{z}}{d r}=-4 \pi \rho v_{\theta} ; \frac{1}{r} \frac{d}{d r} r B_{\theta}=4 \pi \rho v_{z} ; \frac{1}{r} \frac{d}{d r} r E_{r}=4 \pi \rho,
\end{array}
$$

where $v_{\theta}=\omega_{0} r, v_{z}$ are equilibrium velocities, $\omega_{0} \geq 0$ is the angular velocity of the beam rotation as a whole, $\rho$ the charge density, $B_{z} \leq 0$ the full longitudinal magnetic field, $E_{r}, B_{\theta}$ are self fields of the beam, $\gamma=1 / \sqrt{1-v_{\theta}^{2}-v_{z}^{2}}$.

The general macroscopic fluid equations describing the equilibrium of a cylindrical beam allow many solutions. Any two functions can be chosen arbitrarily or two additional relations are necessary to close this selfconsistent set of equations that permit to simulate the experimental circumstances. Here we shall follow the approach [1]. It combines a macroscopic cold fluid description and conservation laws which are in use for a microscopic description based on the Vlasov-equation. We assume that full energy of particles conserved

$$
H=\gamma+\phi=\text { const }
$$

(this means the emission of particles from an equipotential cathode) and that the conservation law for the canonical angular momentum is valid

$$
P_{\theta}=r\left(p_{\theta}+A_{\theta}\right)=\text { const },
$$

(this means the conservation of axial symmetry), where $A_{\theta}=$ $A_{\theta}(r)$ at the source.

Three cases, namely, 1) $P_{\theta}=0$, 2) $P_{\theta}=$ const, and 3) $P_{\theta}=B_{0} r^{2} / 2$ allow to simulate three cases of emission, namely, 1) magnetically shielded source, 2) hollow beam formation launched from a cathode surface coinciding with a magnetic flux surface, and 3) immersed source with a cathode immersed in the longitudinal magnetic field. These two physical relations allow to derive the next general set of equations [1]

$$
\begin{aligned}
\left(r \gamma^{2} v_{z}^{\prime}\right)^{\prime}+\gamma\left(\frac{v_{\theta} P_{\theta}^{\prime}}{v_{z}}\right)^{\prime} & =0, \\
\left(r \gamma^{2} v_{\theta}^{\prime}\right)^{\prime}-\frac{\gamma^{2} v_{\theta}}{r}-r \gamma\left(\frac{P_{\theta}^{\prime}}{r}\right)^{\prime} & =0,
\end{aligned}
$$

where the prime denotes $d / d r$. For the first two cases we get

$$
r \gamma^{2} v_{z}^{\prime}=\text { const } .
$$

The constant equals zero (i.e., $v_{z}=$ const) in the first case, which corresponds to the model of a solid beam emitted from a shielded source and in the second case, which corresponds to a foilless magnetic-insulation diode under the conditions of no central conductor and no axial current inside the drift region. General analytical solutions in these two cases were found in [2] and later in [1]. We do not reproduce them here. Note that in the second case the general solution was represented by Jakobyfunctions. Simplified solution in a case of practical interest, namely, thin-walled annular beam, can be represented by simple functions [3], which is useful for investigating wave processes.

For an immersed source $P_{\theta}$ depends on the radius, as $A_{\theta}$ varies across the emitting surface. Unfortunately, it is impossible to consider the transient region between the cathode and the drift region in the case. We can assume that there is no crossing of trajectories and that radius $r_{c}$ from which an electron is launched at the cathode and radius $r$ of this electron inside the drift region satisfy the simple relation $r=\alpha r_{c}$, where $\alpha$ is constant.

It can be shown that for this case equilibria with $v_{z}=$ const or $\omega_{0}=$ const are impossible. Moreover, the approximate solution shows that due to beating no homogeneity along $\mathrm{z}$ stationary 
states exists if the beam is launched in a drift region with smaller magnetic field than in the cathode region. The nature of these beats is the interaction of the beam with the $B_{r}$ - component of the field. Fast cyclotron rotation of an electron around a force line of the magnetic field is affected by the rotation of the electron under the action of the $B_{r}$ - component, which is paramagnetic in a decreasing field and diamagnetic in an increasing field. Inside a drift region the beam as a whole always rotates diamagnetically and the rotation induced in a decreasing field produces mismatching effects, which are seen as free oscillations of the beam envelope.

Thus, the general approach allows to simulate conditions of beam generation in high-current accelerators and provide the basis for analytical investigation of wave processes in the frame of stringent selfconsistent equilibria at least in two cases: a solid beam $\left(P_{\theta}=0\right)$ and a hollow beam $\left(P_{\theta}=\right.$ const $)$. It is rather difficult to use the model of an immersed beam, which is of practical interest due to the absence an analytical solution. It is shown below that an ordinary simplification that could allow to find an analytical solution leads to the loss of necessary information about the equilibrium state and the same result in this approximation can describe initially different equilibria.

Moreover, we do not consider here a transition region that can have fantastic geometry. The comparison of analytical and numerical results with the results of computer simulation for real geometry shows that to form a beam with parameters close to those for given model, the form of the magnetic field in the transition region must be rather complicated and precise.

\section{GENERAL WAVE EQUATION IN}

\section{ELECTROSTATIC APPROXIMATION}

The electrostatic approximation is used rather often to investigate electrostatic instabilities and slow waves. It is assumed that $\vec{B}=\nabla \times \vec{E} \approx 0$ and $\vec{E}=-\nabla \phi=\{-\partial \phi / \partial r, i m \phi / r, i k z\}$ (all small pertuberation is considered as $\propto \exp (i \omega t-i k z-i m \theta)$ where $\omega$ is the frequency, $\mathrm{k}$ the longitudinal wave number and $\mathrm{m}$ the number of azimuthal variations).

Here, the general wave equation in the electrostatic approximation is presented and completes the general steady state equations [1]. Let us suppose that the conservation law (1) is valid and no additional assumption is used, i.e., we consider a rather wide class of equilibrium states of monoenergetic beams.

Performing routine procedures to linearize the complete set of equations, the wave equations in our case may be written in the form

$$
\begin{array}{r}
\frac{1}{r} \frac{\partial}{\partial r} r L^{2}\left(1+\frac{\omega_{p}^{2} \gamma_{0}}{F_{5}-\gamma_{0}^{2} L^{2}}\right) \frac{\partial \Phi}{\partial r}- \\
\Phi\left\{\left(\frac{m^{2}}{r^{2}}+k^{2}\right)\left(L^{2}-\frac{\omega_{p}^{2}}{\gamma_{0}}\right)+\frac{\omega_{p}^{2}}{\gamma_{0}}\left(\frac{m}{r} v_{\theta}+k v_{z}\right)^{2}+\right. \\
\frac{\omega_{p}^{2}}{\gamma_{0}} \frac{\left(\frac{m}{r} F_{1}+k F_{2}\right)^{2}}{F_{5}-\gamma_{0}^{2} L^{2}}-\frac{L}{r} \frac{\partial}{\partial r} r \frac{\partial L}{\partial r}+ \\
\left.\frac{1}{r} \frac{\partial}{\partial r} r \frac{\omega_{p}^{2} L\left(\frac{m}{r} F_{1}+k F_{2}\right)}{F_{5}-\gamma_{0}^{2} L^{2}}\right\}=0,
\end{array}
$$

where $\Phi=\phi / L, L=\omega-k v_{z}-m v_{\theta} / r$. Functions $F_{i}$ describe an equilibrium state and take the forms

$$
\begin{aligned}
& F_{1}=B_{z 0}+\left(v_{\theta} \gamma_{0}\right)^{\prime}+v_{\theta} \gamma_{0} / r=P_{\theta}^{\prime} / r \\
& F_{2}=\left(v_{z} \gamma_{0}\right)^{\prime}-B_{\theta 0}=-\left(P_{\theta}^{\prime} / r\right) v_{\theta} / v_{z} \\
& F_{3}=F_{1}-\gamma_{0}\left(v_{\theta}^{\prime}-v_{\theta} / r\right) \\
& F_{4}=F_{2}-v_{z}^{\prime} \gamma_{0} \\
& F_{5}=F_{1} F_{3}+F_{2} F_{4},
\end{aligned}
$$

and $v_{\theta} F_{1}+v_{z} F_{2} \equiv 0$. Eqn. (5) is of general form for the given class of equilibrium states and is valid for solid as well as for hollow beams [4].

Taking into account the second conservation law (2), which was used in the first part, one can redefine functions $F_{i}$ by means of $P_{\theta}$. These expressions are in the right-hand member of equations (6).

Equation (5) has its simplest form under the assumption that $P_{\theta}=$ const, which corresponds to the $v_{z}=$ constant class (solid beam launched from shielded source and hollow beam launched from foilless magnetic insulation diode). In the case

$$
F_{1} \equiv F_{2} \equiv F_{4} \equiv F_{5} \equiv 0 ; F_{3}=-\gamma_{0}\left(v_{\theta}^{\prime}-\frac{v_{\theta}}{r}\right)
$$

and equation (5) has the form

$$
\begin{gathered}
\frac{1}{r} \frac{\partial}{\partial r} r\left(L^{2}-\frac{\omega_{p}^{2}}{\gamma_{0}}\right) \frac{\partial \Phi}{\partial r}-\Phi\left\{\left(\frac{m^{2}}{r^{2}}+k^{2}\right)\left(L^{2}-\frac{\omega_{p}^{2}}{\gamma_{0}}\right)+\right. \\
\left.\frac{\omega_{p}^{2}}{\gamma_{0}}\left(\frac{m}{r} v_{\theta}+k v_{z}\right)^{2}-\frac{L}{r} \frac{\partial}{\partial r} r \frac{\partial L}{\partial r}\right\}=0
\end{gathered}
$$

It is an obvious nonessential simplification due to transition to the $v_{z}=0$ system.

When the beam is launched from an immersed source in magnetic field $B_{0}$ we get

$$
\begin{array}{r}
F_{1}=B_{0} ; F_{2}=-B_{0} \frac{v_{\theta}}{v_{z}} ; \\
F_{5}=B_{0}\left[B_{0}\left(1+\frac{v_{\theta}^{2}}{v_{z}^{2}}\right)-\gamma_{0} \frac{v_{z}}{r}\left(\frac{r v_{\theta}}{v_{z}}\right)^{\prime}\right] .
\end{array}
$$

In this case the analytical solution can be found only under special approximations, for example, small currents limit and large external magnetic field. Numerical solutions of steady state equations show that under conditions $B_{z e} r_{e} \geq 1$ and $I \leq 0.1$ transverse distributions of the main interesting parameters $\left(\omega_{p}^{2}, v_{z}, B_{z}, \omega_{0}, L, \gamma\right)$ are close to uniform except $v_{\theta} \propto r$. Note that $B_{0}$ at the cathode differs from $B_{z}$ in the drift region. Using this approximate solution, one can write functions $F_{i}$ as

$$
\begin{aligned}
& F_{1} \approx \omega_{v} ; \quad F_{2} \approx-\left(\omega_{0} B_{0} / v_{z i}\right) ; \\
& F_{3} \approx F_{1} ; \quad F_{4} \approx 0 ; \quad F_{5} \approx \omega_{v}^{2},
\end{aligned}
$$

where $\omega_{v}=B_{z i}+2 \omega_{0} \gamma_{i}, \omega_{0}=-\left(B_{z i}-B_{0}\right) / 2 \gamma_{i}$. Index 'i' signifies axis value. Wave equation (5) can be written as follows

$$
\begin{array}{r}
\frac{1}{r} \frac{\partial}{\partial r} r \frac{\partial \phi}{\partial r}-\phi\left\{\frac{m^{2}}{r^{2}}+\right. \\
\frac{\omega_{v}^{2}-\gamma^{2} L^{2}}{\omega_{v}^{2}-\gamma^{2} L^{2}+\omega_{p}^{2} \gamma}\left(k^{2}\left(1-\frac{\omega_{p}^{2}}{\gamma L^{2}}\right)+\right. \\
\left.\left.\frac{\omega_{p}^{2}}{\gamma L^{2}}\left(m \omega_{0}+k v_{z}\right)^{2}\right)\right\}=0 .
\end{array}
$$


Note that this equation where an approximate steady state solution of 1-st order was used, is valid at least for two other equilibria, namely 1) monoenergetic beam with $\rho=$ const, 2) rigid rotor equilibrium with $v_{z}=$ const. That is, for such a 'universal' approximation all information about special characteristics of the equilibrium was lost. It would be not surprising that in the wave 'language' all these equilibria are the same.

For this simplification, the wave equation for a beam launched from a shielded cathode will be similar to (10) if $\omega_{v} \equiv 0$ and differs qualitatively.

\section{IY. ELECTROMAGNETIC WAVE EQUATION}

\section{FOR A HOLLOW THIN-WALLED BEAM}

It is difficult to find the electromagnetic wave equation in general form due to the cumbersome expressions. But, it is possible to find rather simple equations for a solid beam with $P_{\theta}=0$, at least in the small current limit (of little practical interest) and for a high-current hollow ( $P_{\theta}=$ const), thin-walled beam, which might be useful because of practical interest.

For a thin-walled hollow beam but with large (relativistic) variation of $v_{\theta}$ in cross section under assumptions $d / d r \gg 1 / R$, where $\mathrm{R}$ is the average radius of the beam, $m / R \approx$ const, and $\omega_{p}^{2} / \gamma_{0} \approx$ const, though $\omega_{p}^{2}$ and $\gamma_{0}$ do strongly vary in cross section [3]. In the $v_{z}=0$ system, the result may be written in the form (here $\gamma_{0}=\gamma_{\perp}$ )

$$
\begin{array}{r}
W_{\theta z}^{\prime \prime}+\chi^{2} W_{\theta z}=0 \\
\left(\left(L^{2} \gamma_{0}^{2}-\frac{\omega_{p}^{2}}{\gamma_{0}}\right) W_{\theta}^{\prime}\right)^{\prime}+ \\
\left(L^{2} \gamma_{0}^{2}-\frac{\omega_{p}^{2}}{\gamma_{0}}\right)\left(\chi^{2}+\frac{\omega_{p}^{2}}{\gamma_{0}}\right) W_{\theta}=0 ;
\end{array}
$$

where $\chi^{2}=\omega^{2}-k^{2}-m^{2} / R^{2}-\omega_{p}^{2} / \gamma_{0} \approx$ const, $L=L\left(v_{\theta}\right)$, and

$$
\begin{array}{r}
W_{\theta z}=\left(\chi^{2}+k^{2}\right) E_{z}+k\left(\frac{m}{r}-\frac{\omega_{p}^{2} v_{\theta}}{\gamma_{0} L}\right) E_{\theta} \\
W_{\theta}=\frac{E_{\theta}}{\left(\chi^{2}+k^{2}\right) \gamma_{0} L} .
\end{array}
$$

\section{CONCLUSION}

A general wave equation in the electrostatic approximation, which is valid in any selfconsistent equilibrium, was derived under the only assumption of constancy of full particle energy. This equation supplements general steady state equation and allows to consider different equilibria and their wave properties in closed manner. Equations (5) and (11) allow to investigate and compare properties of electrostatic and electromagnetic waves and instabilities in REB for different equilibrium states corresponding to experimental condition of beam generation in high-current accelerators. These equations permit to compare the influence of different cross terms that can be omitted because of approximate solution of the steady state problem especially for resonant cases.

\section{REFERENCES}

[1] M. Raiser, "Laminar-flow equilibria and limiting currents in magnetically focused relativistic beams", Phys. Fluids 20 (1977) 477.

[2] A. V. Agafonov, V. S. Voronin, A. N. Lebedev, K. N. Pazin, "High-current electron beam transport by means of magnetic field", Sov. JTP 44 (1974) 1909.

[3] A.V.Agafonov, A.N.Lebedev, "Stability of an electron beam with $v / \gamma>1$ in a magnetic field", Sov. JTP 47 (1977) 1729.

[4] A.V. Agafonov, "Equilibria and waves in high-current electron beams emitted from shielded and immersed cathodes". Proc. 10th International Conference on High Power Particle Beams. June 20-24, 1994, San Diego, USA. Vol. 2, p. 511. 